\title{
Removing zero Lyapunov exponents
}

\author{
ALEXANDRE T. BARAVIERA $\uparrow$ and CHRISTIAN BONATTI $\ddagger$ \\ $\dagger$ Departamento de Matemática, Universidade Federal do Rio Grande do Sul, \\ Porto Alegre, RS, Brasil \\ (e-mail: baravi@mat.ufrgs.br) \\ $\$$ Institut de Mathématique de Bourgogne, UMR 5584 du CNRS, BP 47870 , \\ 21078 Dijon Cedex, France \\ (e-mail: bonatti@u-bourgogne.fr)
}

(Received 21 November 2002 and accepted in revised form 16 January 2003)

\begin{abstract}
In an explicit family of partially hyperbolic diffeomorphisms of the torus $T^{3}$, Shub and Wilkinson recently succeeded in perturbing the Lyapunov exponents of the center direction. We present here a local version of their argument, allowing one to perturb the center Lyapunov exponents of any partially hyperbolic system, in any dimension and with arbitrary dimension of the center bundle.
\end{abstract}

\section{Introduction}

One of the classical problems of the theory of dynamical systems is the understanding of the Lyapunov exponents. From Oseledets' Theorem we know that Lyapunov exponents are defined for almost every point with respect to any given invariant measure, and are independent of the point if moreover the measure is ergodic. Pesin's theory recovers some hyperbolic behavior for the points whose Lyapunov exponents are all non-zero (see for instance $[\mathbf{F H Y}])$. In particular, these points have well-defined unstable and stable invariant manifolds. For these reasons, an ergodic invariant measure $\mu$ is called hyperbolic if all its exponents are different from zero.

On the other hand, the presence of zero exponents creates many obstacles to a good ergodic description of the system and is often related to some pathologies. For this reason, it is important to understand in which situations the zero exponents could be removed by perturbations.

In the negative direction a recent result by Bochi [Boc] shows that, for $C^{1}$-generic nonAnosov conservative diffeomorphisms on compact surfaces, almost every point has zero Lyapunov exponents. For a higher dimensional version see [BocVi]. We hope that this kind of result is typical only of the $C^{1}$-topology and that zero Lyapunov exponents are no longer generic for more regular systems. It is possible to illustrate this contrast in the a priori simpler case of linear cocycles: from Bochi we know that zero exponents are generic for non-hyperbolic continuous cocycles of $S L(2, \mathbb{R})$ but this is no longer true assuming that the cocycle satisfies a Hölder condition; see [BoVi], [BoGVi]. 
Our motivation is a very surprising result by Shub and Wilkinson [ShWi]. They deal with a conservative system on the torus $T^{3}$ that is a skew product of an Anosov diffeomorphism of the torus $T^{2}$ by rotations. This dynamics is partially hyperbolic, the center foliation being defined by a fibration $\pi: T^{3} \rightarrow T^{2}$, where the fibers are circles. This fibration, by general results from the theory of partial hyperbolicity, persists under perturbations. Shub and Wilkinson build a conservative perturbation of this system (by a smooth arc) in order to create positive exponents in the direction of the center leaves for Lebesgue almost every point; it seems a priori impossible because the center leaves are compact curves with bounded length, and so each center leaf cuts the set of points with non-zero center Lyapunov exponents in a zero measure set (for the Lebesgue measure of the leaf). The apparent contradiction disappears when one realizes that in fact the new center foliation has a non-absolutely continuous holonomy.

Before stating our results we need to recall some definitions and elementary properties of partially hyperbolic diffeomorphisms.

0.1. Partial hyperbolicity. Let $f$ be a diffeomorphism on a compact manifold $M$ and denote by $f_{*}$ the differential of $f$. An $f_{*}$-invariant splitting $T M=E_{1} \oplus \cdots \oplus E_{k}$ is called a dominated splitting if each $E_{i}$ is a continuous $f_{*}$-invariant sub-bundle of $T M$ and if there is some integer $n>0$ such that, for any $x \in M$, any $i<j$ and any non-zero vectors $u \in E_{i}(x)$ and $v \in E_{j}(x)$, one has

$$
\frac{\left\|f_{*}^{n}(u)\right\|}{\|u\|}<\frac{1}{2} \frac{\left\|f_{*}^{n}(v)\right\|}{\|v\|} .
$$

One of the bundles $E_{i}$ of a dominated splitting is uniformly contracting (respectively uniformly expanding) if, up to increasing the integer $n$ above, one has $\left\|f_{*}^{n}(u)\right\| \leq \frac{1}{2}\|u\|$ (respectively $\left\|f_{*}^{n}(u)\right\| \geq 2\|u\|$ ) for any vector $u \in E_{i}$. We say that $f$ is partially hyperbolic if it admits a dominated splitting $T M=E^{s} \oplus E^{c}$ or $T M=E^{c} \oplus E^{u}$ or $T M=E^{s} \oplus E^{c} \oplus E^{u}$, where $E^{s}$ and $E^{u}$ denote uniformly contracting and expanding sub-bundles, respectively.

Recall that the properties of having a dominated splitting or of being partially hyperbolic are $C^{1}$-open properties, and the bundles of the splitting depend continuously on the diffeomorphism.

Consider now a Riemannian metric on $M$. This metric allows us to define the Jacobian of $f$ on the bundle $E_{i}$, denoted by $J_{f}^{i}(x)$, as the modulus of the determinant of the restriction of $f_{*}(x)$ to $E_{i}(x)$.

If $f$ leaves invariant some ergodic measure $\omega$, then the Lyapunov exponents of $f$ are well defined and constant $\omega$-almost everywhere, so that one can speak of the exponents of $f$ for $\omega$. Moreover, the Lyapunov spaces, at each point where they are defined, are contained in the spaces $E_{i}(x)$; this allows us to speak of the Lyapunov exponents in restriction to the sub-bundle $E_{i}$.

Remark 0.1. If $\omega$ is an $f$-invariant ergodic measure for $f$ and if $T M=E_{1} \oplus \cdots \oplus E_{k}$ is a dominated splitting of $f$, then for each $i$ the sum $\Lambda\left(f, \omega, E^{i}\right)$ of the Lyapunov exponents of $\omega$ in restriction to $E_{i}$ is well defined and is equal to $\int_{M} \log J_{f}^{i}(x) d \omega$. 
Since the sub-bundle $E_{i}$ varies continuously with $f$ one gets that the function $J_{f}^{i}(x)$ also depends continuously on $f$ and so does $\Lambda\left(f, \omega, E^{i}\right)$.

0.2. Statement of the results. Let $M$ be a compact manifold and $\omega$ some volume form on $M$. We say that a $C^{2}$-diffeomorphism $f$ is $C^{r}$-stably ergodic for $\omega$ (with $r=1,2$ ) if it preserves $\omega$ and if $\omega$ is ergodic for any $\omega$-preserving $C^{2}$-diffeomorphism $g$ in a $C^{r}$-neighborhood of $f$.

THEOREM 1. Let $M$ be a compact manifold and $\omega$ be a smooth volume form on $M$. Assume that $f$ is an $\omega$-preserving $C^{1}$-stably ergodic diffeomorphism of $M$, and assume that $f$ admits a dominated splitting of the following type: $E^{s} \oplus E^{c} \oplus E^{u}$, where the subbundles $E^{s}$ and $E^{u}$ are uniformly contracting and expanding, respectively.

Then there is a $C^{2}$-diffeomorphism $g$ arbitrarily $C^{1}$-close to $f$ for which the sum $\Lambda^{c}(g, \omega)$ of the center Lyapunov exponents (that is corresponding to $E_{g}^{c}$ ) of $\omega$ is non-zero.

Using Remark 0.1 above and Theorem 1 one obtains the following.

COROLlARY 0.2. The set of diffeomorphisms whose sum of the center Lyapunov exponents (for $\omega$ ) is non-zero contains a $C^{1}$-dense open subset of the (open) set of partially hyperbolic diffeomorphisms which are $C^{1}$-stably ergodic for $\omega$.

The existence of an open set of $C^{2}$-partially hyperbolic diffeomorphisms which are $C^{1}$-stably ergodic for $\omega$ is assured by recent work of Dolgopyat and Wilkinson [DoWi, Corollary 0.5].

Consider any $C^{r}$ Riemannian metric on $M$. As $f$ is $C^{1}$-stably ergodic, for any $C^{1}$-perturbation $g$ of $f$, the sum $\Lambda^{c}(g, \omega)$ of the center exponents of $g$ for $\omega$ is given by

$$
\Lambda^{c}(g, \omega)=\int_{M} \log J_{g}^{c}(x) d \omega(x)
$$

where $J_{g}^{c}$ is the absolute value of the determinant of the restriction of $D g$ to the center bundle of $g$, for the Riemannian metric on $M$.

So Theorem 1 is a direct consequence of the following.

Proposition 0.3. Let $(M, \omega)$ be a compact manifold endowed with a $C^{r}$ volume form, $r \geq 2$. Let $f$ be a $C^{1} \omega$-preserving diffeomorphism of $M$, admitting a dominated splitting $T M=E^{s} \oplus E^{c} \oplus E^{u}$, where $E^{u}$ is non-trivial and is uniformly expanding, and $E^{s}$ (maybe trivial) is uniformly contracting.

Then there are arbitrarily small $C^{1}$-perturbations $g$ of $f$ such that

$$
\int_{M} \log J_{g}^{c}(x) d \omega(x)>\int_{M} \log J_{f}^{c}(x) d \omega(x) .
$$

In the result above we asked that the bundles $E^{s}$ and $E^{u}$ be uniformly contracting and expanding, respectively, because we were a priori interested in partially hyperbolic diffeomorphisms. However, this property is not used in any argument of the proof: the only property used is the domination. So our arguments actually prove the following. 
THEOREM 2. Let $M$ be a compact manifold and $\omega$ be a smooth volume form. Let $f$ be an $\omega$-preserving $C^{1}$-diffeomorphism of $M$, admitting a dominated splitting $T M=$ $E_{1} \oplus \cdots \oplus E_{k}, k>1$.

Then there are $\omega$-preserving diffeomorphisms $g$, arbitrarily $C^{1}$-close to $f$, for which the integral $\int_{M} \log J_{g}^{i}(x) d \omega(x)$ is different from 0 for each $i \in\{1, \ldots, k\}$.

Remark 0.4. Our local argument above has allowed us to deal with a large class of diffeomorphisms. This generality has a cost: we lost some smallness and regularity of the perturbation. In [ShWi] the perturbation inducing the positive center exponent is obtained by a smooth arc of diffeomorphisms $f_{t}$ such that $f_{0}=f$ and any $f_{t}, t \neq 0$, has positive center Lyapunov exponent (in fact the proof in [ShWi] consists in controlling the second derivative of the unstable Lyapunov exponent with respect to the parameter). Here, we produce an arc of $C^{r}$-diffeomorphisms, converging to $f$ in the $C^{1}$-topology when the parameter goes to 0 , but the arc is not differentiable at the parameter 0 . Furthermore, our general argument does not work in a $C^{r}$-topology when $r>1$.

0.3. An application. We finish this section by giving an example where Theorem 1 applies.

Let $M$ be a compact manifold endowed with a $C^{2}$-volume form $\omega$ and let $X$ be a $C^{1}$, volume-preserving Anosov flow of $M$. Let $f$ be the time-one map of $X$ : it is a $C^{1}$-partially hyperbolic diffeomorphism whose center bundle is directed by $X$, that is $E^{c}=\mathbb{R} X$.

Then Proposition 0.3 implies that there is an $\omega$-preserving diffeomorphism $g$ arbitrarily $C^{1}$-close to $f$ such that $\int_{M} \log J_{g}^{c}(x) d \omega(x)>0$. The theory of partial hyperbolic systems, see [HPS], implies that any diffeomorphism $g$ sufficiently $C^{1}$ close to $f$ admits a center foliation $\mathcal{F}_{g}^{c}$ conjugated to the center foliation of $f$. Moreover each leaf of $\mathcal{F}_{g}^{c}$ is $g$-invariant and the length $d_{c}(x, g(x))$ of the smaller center segment joining $x$ to $g(x)$ is uniformly bounded by some constant $d$. Then

Proposition 0.5. For any diffeomorphism $g C^{1}$-close to $f$ and any leaf $L_{c}$ of $\mathcal{F}_{g}^{c}$, the set of points of $L_{c}$ having positive Lyapunov exponents has Lebesgue measure 0 in $L_{c}$.

Proof. This is a consequence of the following argument (due to Mañe): if some leaf $L_{c}$ intersects the set with center positive (non-zero) exponents in a set of positive Lebesgue measure (equivalent to the arc length in $L_{c}$ ) then the leaf must be exponentially expanded by the map. But this is not possible because the center leaves can grow at most in a linear way: the distance between $x$ and $g^{n}(x)$ along the center leaf through $x$ is at most $n d$.

As a consequence, and as in [ShWi], we get that $\mathcal{F}_{g}^{c}$ is not absolutely continuous with respect to Lebesgue for any $\omega$-preserving $g$ close to $f$ such that $\int_{M} \log J_{g}^{c}(x) d \omega(x)>0$.

We can strengthen the results if the diffeomorphism is stably ergodic, since in this case it follows that not only the integral of the center exponent is positive, but the exponent itself is positive on a full measure set (i.e. $\omega$-almost everywhere). From Theorem 1 and the paper by Dolgopyat and Wilkinson [DoWi, Corollary 0.5] we can obtain an open set with ergodic diffeomorphisms. 
COROllary 0.6. Let $f$ be the time-t map, $t \neq 0$, of an $\omega$-preserving Anosov flow. There exists a $C^{1}$-open neighborhood $\mathcal{U} \subset \mathcal{P} \mathcal{H}_{\omega}^{2}(M)$ (the set of partially hyperbolic $C^{2}$ diffeomorphisms leaving the measure $\omega$ invariant) of $f$ and $a C^{1}$-open and dense subset $\mathcal{U}_{0} \subset \mathcal{U}$ in which any diffeomorphism is stably ergodic and the Lyapunov center exponent of $\omega$ is not zero.

\section{A three-dimensional case}

In this section, in order to present the idea of the proof of Proposition 0.3 in a clearer setting, we assume very strong simplifying hypotheses (which will be removed in the next section):

- $\quad M$ is a compact 3-manifold endowed with a $C^{2}$-volume form $\omega$.

- $f$ is an $\omega$-preserving diffeomorphism of $M$.

- $f$ leaves invariant a dominated splitting $T M=E^{s} \oplus E^{c} \oplus E^{u}$ where all the bundles are one-dimensional.

- $\quad f$ admits a non-periodic orbit $p_{0}$ and $C^{1}$-local coordinates $(x, y, z)$ centered at $p$ such that the expression of $\omega$ in these coordinates coincides with Lebesgue measure on $\mathbb{R}^{3}$, and the bundles $E^{s}, E^{c}$ and $E^{u}$ are directed by $\partial / \partial x, \partial / \partial y$ and $\partial / \partial z$, respectively. This condition implies the integrability of the bundles $E^{s} \oplus E^{u}, E^{s} \oplus E^{c}$, $E^{c} \oplus E^{u}$, which is not generic. This condition will simplify the argument, but is not essential to it, as will be shown in the next section.

Remark 1.1. These hypotheses are satisfied by the time one map of the vector field obtained by suspending a linear Anosov map $A$ of the torus $T^{2}$, and also by any map obtained as the product of $A$ of $T^{2}$ by a rotation of the circle $S^{1}$.

Let $V$ be a neighborhood of $p_{0}$ where the local coordinates $(x, y, z)$ are defined, endowed with the Euclidean metric. For any $r>0$ small enough, we denote by $B_{r}$ the ball of radius $r$ (for this metric) centered at $p_{0}$.

Let $B(0,1)$ be the unit ball of $\mathbb{R}^{3}$, and denote by $\mu$ the Lebesgue measure on $B(0,1)$. Given any ball $B_{r} \subset V$ we denote by $\varphi_{r}: B_{r} \rightarrow B(0,1)$ the diffeomorphism whose expression in the coordinates is a homothety of ratio $1 / r$. Given any volume-preserving diffeomorphism $h$ of $B(0,1)$ coinciding with the identity map on a neighborhood of the boundary $S(0,1)=\partial B(0,1)$, we denote by $h_{r}$ the diffeomorphism of $M$ with support in $B_{r}$ which coincides with $\varphi_{r}^{-1} \circ h \circ \varphi_{r}$ on $B_{r}$.

We consider a volume-preserving diffeomorphism $h$ of $B(0,1)$ coinciding with the identity map on a neighborhood of $S(0,1)$ and preserving the first coordinate $x$.

THEOREM 3. For any such $h, C^{1}$-close enough to the identity and different from the identity map, one has

$$
\int_{M} \log J_{f_{r}}^{c}(x) d \omega(x)>\int_{M} \log J_{f}^{c}(x) d \omega(x)
$$

for any $r>0$ small enough, where $f_{r}=f \circ h_{r}$.

1.1. A local estimate. Let us write $h(p)_{*}(\partial / \partial z)=h^{u}(p) \partial / \partial z+h^{c}(p) \partial / \partial y$.

We assume that $\|h-i d\|_{\mathrm{C}^{1}}<1$ so that $h^{u}(p)>0$ for any $p \in B(0,1)$. 
LEMMA 1.2. With the notations above, if $h$ is not the identity map then

$$
\int_{B(0,1)} \log h^{u}(p) d \mu(p)<0
$$

Proof. Given any vertical segment $\gamma$ (i.e. tangent to the $z$ direction), we will first prove that the integral of $h^{u}$ along $\gamma$ is non-positive. Denoting by $l(\gamma)$ the arc-length of the curve $\gamma$ we have, since $h$ is the identity close to the boundary of the ball, that

$$
\int_{\gamma} h^{u}(\gamma(\zeta)) \frac{d \zeta}{l(\gamma)}=1
$$

Then, since the logarithm is convex, it is possible to use the Jensen inequality to show that

$$
l(\gamma) \int_{\gamma} \log h^{u}(\zeta) \frac{d \zeta}{l(\gamma)} \leq l(\gamma) \log \int_{\gamma} h^{u}(\zeta) \frac{d \zeta}{l(\gamma)}=0 .
$$

To see that the inequality is in fact strict we need to exhibit at least one point where $h^{u}$ is less than one. Assume by contradiction that $h^{u}$ is constant equal to 1 ; as $h$ coincides with the identity close to the boundary, this implies that the coordinate $z$ is preserved by $h$. Recall that $h$ preserves the coordinate $x$, so it leaves invariant each segment $\sigma_{x_{0}, z_{0}}=\left\{(x, y, z) \subset B(0,1) \mid x=x_{0}\right.$ and $\left.z=z_{0}\right\}$. Furthermore, $h$ coincides with the identity map in a neighborhood of the boundary: so in any segment $\sigma$ on which $h$ is not the identity, there is a point where the derivative (in restriction to $\sigma$ ) is less than 1 , contradicting the fact that $h$ preserves the volume.

We denote $I(h)=\int_{B(0,1)} \log h^{u}(p) d \mu(p)$.

1.2. Perturbation of the action of $f$ on the unstable direction. We fix some $h$ as in the subsection above and for any ball $B_{r}$ we denote $f_{r}=f \circ h_{r}$. The aim of this section is to describe the integral of the logarithm of the unstable Jacobian of $f_{r}$. The difficulty here is the control of the unstable direction, since it obviously changes after the perturbation. We bypass this problem by substituting the true dynamics $f_{r}$ by some linear cocycle having the same Lyapunov exponent for Lebesgue almost every point. Let us now describe this cocycle.

We consider the one-dimensional fiber sub-bundle $E_{f}^{u}$ of $T M$ which is the unstable bundle of $f$. For notational simplicity we write $u(p)$ for the unit vector generating $E_{f}^{u}(p)$. Notice that this sub-bundle is not invariant by $f_{r}$ (but is invariant by $f$ ). We now describe an action $F_{r}$ of $f_{r}$ on $E_{f}^{u}$, that is, for every $p \in M, F_{r}(p): E_{f}^{u}(p) \rightarrow E_{f}^{u}\left(f_{r}(p)\right.$ is a linear map defined as follows.

- The action of $F_{r}$ coincides with the action of $f_{*}$ (we recall that $f_{*}$ is the differential of $f$ ) for all points outside the set $B_{r} \cup f^{-1}\left(B_{r}\right)$ (which is possible because $f_{r}$ coincides with $f$ so that $E_{f}^{u}$ is $f_{r}$-invariant on this set) since we would like to keep as much as possible the information about the expansiveness of $f$.

- In $B_{r}$, the action of $F_{r}$ is the action of $f_{*}$ on the projection of $\left(h_{r}\right)_{*} u$ on $E_{f}^{u}$ parallel to the center bundle $E_{f}^{c}$ of $f$, so that we keep artificially $E_{f}^{u}$ as an invariant direction.

- Consider some point $p \in f^{-1}\left(B_{r}\right)$. If the negative orbit of $p$ (by $f$ or equivalently by $f_{r}$ ) is disjoint from $B_{r}$ then $F_{r}$ coincides with $f_{*}$ at $p$. In the other case let $\tilde{q} \in B_{r}$ 
and $q=h_{r}(\tilde{q}) \in B_{r}$ be the points whose first passage in $f^{-1}\left(B_{r}\right)$ by $f_{r}$ and $f$ respectively is precisely $p$, and denote by $n(p)$ the integer such that $p=f^{n(p)}(q)$.

- $\quad$ The action of $F_{r}$ on $\tilde{q} \in B_{r}$ ignored a small vector $v(q)$ at $q$ in the $E_{f}^{c}$ direction. We consider the image $f_{*}^{n(p)}(v(q))$ and we consider its projection $w(p)$ on $E_{f}^{u}$ parallel to the new center bundle $E_{f_{r}}^{c}$. So $w(p)$ is the correction we have to add to the vector $F_{r}^{n_{p}}(u(\tilde{q})) \in E_{f}^{u}(p)$. We call $A(p)$ the multiplicative effect of this correction. Finally $F_{r}(p)$ is the composition of $f_{*}(p)$ by the homothety of ratio $A(p)$.

In what follows we make this procedure more precise.

For any $p$ we denote $f_{*}(u(p))=\lambda(p) \cdot u\left(f_{r}(p)\right)$ with $\lambda(p)=J_{f}^{u}(p)$, and $F_{r}(p, u(p))=\left(f_{r}(p), \lambda_{r}(p) \cdot u\left(f_{r}(p)\right)\right)$, where $\lambda_{r}(p)$ is defined by:

$$
\lambda_{r}(p)= \begin{cases}\lambda(p), & \text { if } p \notin B \cup f^{-1}(B), \\ \lambda\left(h_{r}(p)\right) h_{r}^{u}(p), & \text { if } p \in B, \quad \text { where } h_{r}^{u}(p)=h^{u}\left(\varphi_{r}(p)\right), \\ \lambda(p) A(p), & \text { if } p \in f^{-1}(B) .\end{cases}
$$

We have to define $A(p)$, for $p \in f^{-1}\left(B_{r}\right)$. For any $p \in f^{-1}\left(B_{r}\right)$ we define $n(p)$ as the least natural number such that $p=f_{r}^{n(p)}(\tilde{q})$ with $\tilde{q} \in B_{r}$ (and $n(p)=+\infty$ if the negative orbit of $p$ is disjoint from $B_{r}$ ). If $n(p)=+\infty$ we choose $A(p)=1$.

Let us write $q=h_{r}(\tilde{q})$, which implies $p=f_{r}^{n(p)}(\tilde{q})$. We define $\left(h_{r}\right)_{*}(u(\tilde{q}))=$ $h_{r}^{u}(\tilde{q}) \cdot u(q)+v(q)$. Then

$$
\left(f_{r}^{n(p)}\right)_{*}(u(\tilde{q}))=h_{r}^{u}(\tilde{q}) \prod_{0}^{n(p)-1} \lambda\left(f^{i}(q)\right) u(p)+f_{*}^{n(p)}(v(q)),
$$

and

$$
\left(F_{r}^{n(p)}\right)(u(\tilde{q}))=h_{r}^{u}(\tilde{q}) \prod_{0}^{n(p)-1} \lambda\left(f^{i}(q)\right) u(p) .
$$

$F_{r}^{n(p)}(u(\tilde{q}))$ is well defined because $F_{r}$ is already defined out of $f^{-1}\left(B_{r}\right)$.

Let $P_{r, p}^{u}$ be the projection of $\left.T M\right|_{p}$ on $E_{f}^{u}(p)$ along the new center bundle. We denote $w(p)=P_{r, p}^{u}\left(f_{*}^{n(p)}(v(q))\right)$.

Then $A(p)$ is the multiplicative correction term we get when we replace $F_{r}^{n(p)}(u(\tilde{q}))$ by the projection $P_{r, p}^{u}\left(\left(f_{r}^{n(p)}\right)_{*}(u(\tilde{q}))\right)$, that is

$$
A(p)=\frac{\left\|P_{r, p}^{u}\left(\left(f_{r}^{n(p)}\right)_{*}(u(\tilde{q}))\right)\right\|}{\left\|F_{r}^{n(p)}(u(\tilde{q}))\right\|}=1+\frac{\langle w(p), u(p)\rangle}{h_{r}^{u}(\tilde{q}) \prod_{0}^{n(p)-1} \lambda\left(f^{i}(q)\right)} .
$$

Notice that $F_{r}$ is a measurable, uniformly bounded linear cocycle over $f_{r}$, which is a conservative diffeomorphism. Consequently, the Lyapunov exponent of the Lebesgue measure is well defined for Lebesgue almost every point for this cocycle. Now let us show that its integral is indeed the unstable exponent of $f_{r}$.

Remark 1.3. Let $m$ be the first return time in $B_{r}$ of a point $p \in B_{r}$. By construction we have that $F_{r}^{m}(u(p))$ is the projection of $\left(f_{r}^{m}\right)_{*}(u(p))$ on the unstable bundle $E_{f}^{u}$ along the new center-stable bundle $E_{f_{r}}^{c s}$. Using the invariance of $E_{f_{r}}^{c s}$ we have that this is true for any future iterate that is a return to the ball. 
LEMMA 1.4. For every point $p$ where the Lyapunov exponents are defined, the Lyapunov exponent of $p$ for $F_{r}$ is the unstable Lyapunov exponent of $p$ for $f_{r}$.

Proof. Let $p$ be some point of $B_{r}$, and $N>0$ such that $f_{r}^{N}(p) \in B_{r}$. Then, by Remark 1.3, $F_{r}^{N}(u(p))$ is just the projection of $\left(f_{r}^{N}\right)_{*}(u(p))$ on $E_{f}^{u}$ along the new center stable bundle.

This bundle is transversal $\dagger$ to $E_{f}^{u}$, and the vector $\left(f_{r}^{N}\right)_{*}(u(p))$ belongs to an unstable cone field whose angle with $E_{f}^{u}$ is uniformly bounded, so that this projection affects the norm in a uniformly bounded way and this correcting term disappears by taking the logarithm and dividing by the iterate. Finally, the vector $\left(f_{r}^{N}\right)_{*}\left(u_{p}\right)$ is transversal to the center stable bundle so that its exponential growth is the unstable Lyapunov exponent.

From the last result we obtain the following.

\section{COROLlary 1.5 .}

$$
\int \log J_{f_{r}}^{u}(p) d \omega(p)=\int \log \left(\lambda_{r}(p)\right) d \omega(p)
$$

To see this, it suffices to note that the first integral corresponds to the integral on the expansive Lyapunov exponent of $f_{r}$ and the second to the exponent of the cocycle, both being the same (when they are defined) in consequence of Lemma 1.4.

1.3. The proof of Theorem 3. Denote by $\sigma^{u}$ and $\sigma_{r}^{u}$ the integral of the unstable Jacobian for $f$ and $f_{r}$, respectively, i.e.

$$
\sigma^{u}=\int \log J_{f}^{u}(p) d \omega(p)=\int \log (\lambda(p)) d \omega(p)
$$

and

$$
\sigma_{r}^{u}=\int \log J_{f_{r}}^{u}(p) d \omega(p)=\int \log \left(\lambda_{r}(p)\right) d \omega(p)
$$

Our aim is to show the following result.

PROPOSITION 1.6. For any small $r>0$ the difference $\sigma^{u}-\sigma_{r}^{u}$ is strictly positive.

First, let us remark that $\lambda(p)=\lambda_{r}(p)$ for $p \notin B \cup f^{-1}(B)$ and then $\sigma^{u}-\sigma_{r}^{u}=\int_{B_{r}}\left[\log (\lambda(p))-\log \left(\lambda_{r}(p)\right)\right] d \omega(p)+\int_{f^{-1} B_{r}}\left[\log (\lambda(p))-\log \left(\lambda_{r}(p)\right)\right] d \omega(p)$.

$\dagger$ Here we use that $h$ is $C^{1}$-close to the identity so that the invariant (stable, unstable and center) bundles of $f_{r}$ are close to the invariant bundle of $f$. 
Notice that

$$
\begin{aligned}
\int_{B_{r}}\left[\log (\lambda(p))-\log \left(\lambda_{r}(p)\right)\right] d \omega(p) \\
\quad=\int_{B_{r}} \log (\lambda(p)) d \omega(p)-\int_{B_{r}} \log \left(\lambda_{r}(p)\right) d \omega(p) \\
=\int_{B_{r}} \log (\lambda(p)) d \omega(p)-\int_{B_{r}} \log \left[\lambda\left(h_{r}(p)\right) h_{r}^{u}(p)\right] d \omega(p) \\
=\int_{B_{r}} \log (\lambda(p)) d \omega(p)-\int_{B_{r}} \log \left[\lambda\left(h_{r}(p)\right)\right] d \omega(p)-\int_{B_{r}} \log \left(h_{r}^{u}(p)\right) d \omega(p) \\
=-\int_{B_{r}} \log \left(h_{r}^{u}(p)\right) d \omega(p) \\
=-\operatorname{vol}\left(B_{r}\right) I(h) .
\end{aligned}
$$

In the same way $\int_{f^{-1}\left(B_{r}\right)}\left[\log (\lambda(p))-\log \left(\lambda_{r}(p)\right)\right] d \omega(p)=-\int_{f^{-1}\left(B_{r}\right)} \log (A) d \omega(p)$. In particular

$$
\left|\int_{f^{-1}\left(B_{r}\right)}\left[\log (\lambda(p))-\log \left(\lambda_{r}(p)\right)\right] d \omega(p)\right| \leq \operatorname{vol}\left(B_{r}\right) \max _{f^{-1}\left(B_{r}\right)} \log (A(p)) .
$$

So we get

$$
\sigma^{u}-\sigma_{r}^{u} \geq \operatorname{vol}\left(B_{r}\right)\left[-I(h)-\max _{f^{-1}\left(B_{r}\right)} \log (A(p))\right] .
$$

Let $n_{r}$ be the least strictly positive integer $n$ such that $f^{n}\left(B_{r}\right) \cap B_{r}$ is not empty.

LEMMA 1.7. There is some $\alpha \in] 0,1[$ and some constant $C>0$ such that, for any $r$, one has

$$
\max _{f^{-1}\left(B_{r}\right)}|\log (A(p))| \leq C \alpha^{n_{r}} .
$$

Proof. It is enough to find some $\alpha$ such that $|A(p)-1| \leq C_{0} \alpha^{n_{r}}$.

Let us use the notation introduced for the definition of $A(p), p \in f^{-1}\left(B_{r}\right)$ : we write $p=f^{n_{p}}(\tilde{q}), q \in B_{r}$ and $q=h_{r}(\tilde{q})$, and notice that $n(p) \geq n_{r}$. Then $\left(h_{r}\right)_{*}(u(\tilde{q}))=$ $h_{r}^{u}(\tilde{q}) \cdot u(q)+v(q)$, and $w(p)$ is the projection $P_{r, p}^{u}\left(f^{n(p)}(v(q))\right.$.

We have to show that

$$
\frac{\|w(p)\|}{h_{r}^{u}(\tilde{q}) \prod_{0}^{n(p)-1} \lambda\left(f^{i}(q)\right)} \leq C_{0} \alpha^{n_{r}} .
$$

By definition of the domination, there are constants $C_{1}>0$ and $\left.\alpha \in\right] 0,1[$ such that for any point $x \in M$, any integer $n \in \mathbb{N}$ and any unit vectors $w_{1} \in E_{f}^{u}(x)$ and $w_{2} \in E_{f}^{c}(x)$, one has $\left\|f_{*}^{n}\left(w_{1}\right)\right\| \leq C_{1} \alpha^{n}\left\|f_{*}^{n}\left(w_{2}\right)\right\|$.

Then

$$
\frac{\left\|f_{*}^{n(p)}(v(q))\right\|}{\prod_{0}^{n(p)-1} \lambda\left(f^{i}(q)\right)} \leq C_{1} \alpha^{n(p)}\|v(q)\| \leq C_{1} \alpha^{n_{r}}\|v(q)\| .
$$

Define $C_{2}=\max \left[\left\|P_{W}(v)\right\| /\|v\|\right]$ where $W$ is a plane in the center-stable cone, $v$ is a non-zero vector in the center-stable cone, and $P_{W}(v)$ is the projection of $v$ on $E_{f}^{u}$ parallel to $W$. So $\|w(p)\| /\left\|f_{*}^{n(p)}(v(q))\right\| \leq C_{2}$. Let $C_{3}$ be the max of $1 / h_{r}^{u}(x)$ for $x \in B$, so that $1 / h_{r}^{u}(\tilde{q}) \leq C_{3}$. Now the inequality (2) follows immediately writing $C_{0}=C_{1} \cdot C_{2} \cdot C_{3}$. 
Proof of Proposition 1.6. Lemma 1.2 asserts that $I(h)<0$. Using (1) and Lemma 1.7, it is enough to find some $r$ such that $C \alpha^{n_{r}}<-I(h)$, that is, such that $n_{r}$ is sufficiently large. As $p_{0}$ is non-periodic, $n_{r}$ goes to $+\infty$ when $r$ goes to 0 .

End of the proof of Theorem 3. Notice that the perturbation $h_{r}$ has been chosen in order to preserve the center-unstable bundle $E_{f}^{c u}$ (recall that $h$ preserves the first coordinate). Moreover, as $h_{r}$ preserves the volume form $\omega$, the center-unstable Jacobian of $h_{r}$ is constant and equal to 1 (for any Riemannian metric on $M$ coinciding with the Euclidean one on the local coordinates we fixed in the neighborhood of $p$ ). So the integral of the logarithm of the center-unstable Jacobian of $f_{r}$ is kept unchanged: it is equal to the one of $f$. Observing that the center-unstable Jacobian is the product of the center Jacobian and the unstable one, one gets that

$$
\begin{aligned}
& \int_{M} \log J_{f_{r}}^{c}(x) d \omega(x)-\int_{M} \log J_{f}^{c}(x) d \omega(x) \\
& \quad=\int_{M} \log J_{f}^{u}(x) d \omega(x)-\int_{M} \log J_{f_{r}}^{u}(x) d \omega(x)=\sigma^{u}-\sigma_{r}^{u}>0 .
\end{aligned}
$$

2. Partially hyperbolic diffeomorphisms: $C^{1}$-perturbations

The aim of this section is to prove Theorem 1 in the following setting.

$M$ is a compact $k$-dimensional manifold endowed with a $C^{1}$-volume form $\omega$ and we denote by $\mathcal{P H}_{\omega}^{1}(M)$ the space (endowed with the $C^{1}$-topology) of the $\omega$-preserving $C^{1}$-diffeomorphisms $f$ of $M$ admitting a dominated splitting $T M=E^{s} \oplus E^{c} \oplus E^{u}$ in three fiber bundles: $E^{s}$ (maybe trivial) is uniformly contracting and $E^{u}$ is non-trivial and uniformly expanding.

We choose a Riemannian metric $\|\cdot\|$ and we denote by $J_{f}^{c}(x)$ the center Jacobian of $f$ at a point $x \in M$ for this metric.

THEOREM 4. There is a dense open subset of $\mathcal{P} \mathcal{H}_{\omega}^{1}(M)$ of diffeomorphisms $f$ for which

$$
\int_{M} \log \left|J_{f}^{c}(x)\right| d \omega(x) \neq 0 .
$$

In fact we will prove Proposition 0.3, that is, for any diffeomorphism $f \in \mathcal{P} \mathcal{H}_{\omega}^{1}(M)$ there are arbitrarily small $\omega$-preserving $C^{1}$-perturbations $g$ of $f$ such that $\dagger$

$$
\int_{M} \log J_{g}^{c}(x) d \omega(x)>\int_{M} \log J_{f}^{c}(x) d \omega(x) .
$$

The proof is almost equal to the proof of Theorem 3: we will present a family of local perturbations $f_{r}$ of $f$ by composing $f$ with a diffeomorphism $h_{r}$ whose support is a ball $B_{r}$ of radius $r$ around a non-periodic orbit $p_{0}$. As in Theorem $3, h_{r}$ is built by conjugacy with a homothety of a diffeomorphism $h$ on the unit ball of $\mathbb{R}^{k}$. Two difficulties appear.

- The first difficulty is that we cannot choose $h_{r}$ in order to preserve exactly the integral of the logarithm of a center-unstable Jacobian (or equivalently of the stable Jacobian: the sum is always 0 , due to the preservation of $\omega$ ). To bypass this difficulty we

$\dagger$ We can see in the proof that the uniform contraction and expansion of the bundles $E^{s}$ and $E^{u}$ is nowhere used. The same argument, just using the domination, decreases $\int_{M} \log J_{f}^{u}(x) d \omega(x)$ and increases $\int_{M} \log J_{g}^{c}(x) d \omega(x)$. 
will perturb the sum $\int_{M} \log \left|J_{f}^{u}(x)\right| d \omega(x)+\int_{M} \log \left|J_{f}^{s}(x)\right| d \omega(x)$ instead of just $\int_{M} \log \left|J_{f}^{u}(x)\right| d \omega(x)$.

- In order to control the action of the perturbation on the unstable Jacobian, as in the proof of Theorem 3 we build a cocycle $F_{r}^{u}$ acting on the unstable bundle of $f$ and which coincides with $f_{*}$ out of $B_{r} \cup f^{-1}\left(B_{r}\right)$. This dissociates the role of the perturbation on $B_{r}$ and on $f^{-1}\left(B_{r}\right)$. The control of the action on $f^{-1}\left(B_{r}\right)$ will be identical as in Theorem 3. However, in Theorem 3, the action on the integral of the unstable Jacobian due to the cocycle restricted to $B_{r}$ was just the product $\operatorname{vol}\left(B_{r}\right) \times I(h)$ : this is no longer the case, but we will show that it remains true 'asymptotically' when $r$ goes to 0 .

2.1. Local perturbations. Let $p_{0}$ be a non-periodic point of $f$ (such a non-periodic point always exists in our context). Let $V$ be an open set containing $p_{0}$. We can consider a local chart $\varphi: V \rightarrow B(0,1) \subset \mathbb{R}^{k}(k=\operatorname{dim} M)$ such that $\varphi(\omega)=$ Leb and that sends the directions $E_{p_{0}}^{s}, E_{p_{0}}^{c}, E_{p_{0}}^{u}$ respectively to $E_{x}=\mathbb{R}^{d_{s}} \times\left\{0_{d_{c}}, 0_{d_{u}}\right\}, E_{y}=\left\{0_{d_{s}}\right\} \times \mathbb{R}^{d_{c}} \times\left\{0_{d_{u}}\right\}$, $E_{z}=\left\{0_{d_{s}}, 0_{d_{c}}\right\} \times \mathbb{R}^{d_{u}}$, where $d_{s}, d_{c}, d_{u}$ are the dimensions of $E_{p_{0}}^{s}, E_{p_{0}}^{c}, E_{p_{0}}^{u}$, respectively. We fix a Riemannian metric on $M$ in such a way that $\varphi$ is an isometry on the Euclidean metric on $B(0,1)$.

As in the first section, we fix a volume-preserving diffeomorphism $h$ of $B(0,1)$ coinciding with the identity in the neighborhood of the boundary $S(0,1)$ and preserving the $d_{s}$ first coordinates $x_{1}, \ldots, x_{d_{s}}$. For any other radius $r$ we can define the function $h_{r}: B(0, r) \rightarrow B(0, r)$ as the conjugation of $h$ by the homothety of ratio $r$. Since there is no confusion, we denote also by $h_{r}$ the map induced on $M$ by $h_{r}$ that is the identity outside $B_{r}=\varphi^{-1}(B(0, r))$ and $\varphi^{-1} \circ h_{r} \circ \varphi$ on $B_{r}$.

Now we denote by $f_{r}=f \circ h_{r}$ the perturbation of the diffeomorphism $f$, which clearly preserves the measure $\omega$.

Then Theorem 1 and Proposition 0.3 are a direct consequence of the following.

Proposition 2.1. For any $r>0$ small enough one has

$$
\begin{aligned}
\int_{M} \log \left|J_{f}^{u}(x)\right| d \omega(x)-\int_{M} \log \left|J_{f_{r}}^{u}(x)\right| d \omega(x) \\
>\left|\int_{M} \log \right| J_{f_{r}}^{s}(x)\left|d \omega(x)-\int_{M} \log \right| J_{f}^{s}(x)|d \omega(x)| .
\end{aligned}
$$

We introduce the notations

$$
\sigma_{r}^{u}=\int_{M} \log \left|J_{f_{r}}^{u}(x)\right| d \omega(x) \quad \text { and } \quad \sigma^{u}=\int_{M} \log \left|J_{f}^{u}(x)\right| d \omega(x) .
$$

In the same way we can also define $\sigma^{s}$ and $\sigma_{r}^{s}$.

2.2. Local estimate. For any $p \in B(0,1) \subset \mathbb{R}^{k}$ we define $h^{u}(p)$ as the Jacobian (modulus of the determinant) of the linear map of $E_{z}$ obtained as the composition of the differential of $h$ at $p$ with the projection of $\mathbb{R}^{k}$ on $E_{z}$ parallel to $E_{x} \oplus E_{y}$. In other words $h^{u}(p)$ is the Jacobian of the matrix $\left(\partial h^{i}(p) / \partial x_{j}\right), i>d_{s}+d_{c}$ and $j>d_{s}+d_{c}$, where $h_{i}$ denotes the $i$ th component of $h$ in canonical coordinates. 
If we define analogously $h^{s}(p)$, our hypothesis that $h$ preserves the $d_{s}$ first coordinates implies that $h^{s} \equiv 1$ on $B(0,1)$.

We define $I^{u}(h)=\int_{B(0,1)} \log h^{u}(p) d \operatorname{Leb}(p)$ and one has:

LEMMA 2.2. For any $h$ such that $h^{u}$ is not identically 1 , one has $I^{u}(h)<0$.

The proof is identical to the proof of Lemma 1.2, so we omit it.

Given a point $p$ in $B_{r}$, let $h_{r}^{u}(p)$ be the Jacobian (modulus of the determinant) of the linear map $H_{r}^{u}(p): E_{f}^{u}(p) \rightarrow E_{f}^{u}\left(h_{r}(p)\right)$ obtained as the composition of the differential $\left(h_{r}\right)_{*}(p)$ with the projection of $T_{h_{r}(p)} M$ on $E_{f}^{u}\left(h_{r}(p)\right)$ parallel to $E_{f}^{c s}\left(h_{r}(p)\right)$. Analogously, we can define the function $h_{r}^{s}(p)$.

LEMMA 2.3. With the above notations we have

$$
\lim _{r \rightarrow 0} \frac{\int_{B_{r}} \log \left(h_{r}^{u}(p)\right) d \omega(p)}{\operatorname{vol}\left(B_{r}\right)}=I(h)
$$

and

$$
\lim _{r \rightarrow 0} \frac{\int_{B_{r}} \log \left(h_{r}^{s}(p)\right) d \omega(p)}{\operatorname{vol}\left(B_{r}\right)}=0 .
$$

Proof. Let $\tilde{E}_{r}^{s}, \tilde{E}_{r}^{c}$ and $\tilde{E}_{r}^{u}$ be the bundles on $B(0,1)$ obtained as the image of the bundles $E^{s}, E^{c}$ and $E^{u}$ on $B_{r}$ by the composition of $\varphi$ by the homothety of ratio $1 / r$.

Using the action of $h$ on these bundles, one defines (in the same way as above) functions $\tilde{h}_{r}^{s}$ and $\left.\tilde{h}_{r}^{u}: B(0,1) \rightarrow\right] 0,+\infty\left[\right.$. As $\varphi$ is an isometry we get that $\tilde{h}_{r}^{u}(x)=h_{r}^{u}\left(\varphi^{-1}(x / r)\right)$ and $\tilde{h}_{r}^{s}(x)=h_{r}^{s}\left(\varphi^{-1}(x / r)\right)$. So,

$$
\int_{B(0,1)} \log \left(\tilde{h}_{r}^{u}(p)\right) d \mu(p)=\frac{\int_{B_{r}} \log \left(h_{r}^{u}(p)\right) d \omega(p)}{\operatorname{vol}\left(B_{r}\right)}
$$

and

$$
\int_{B(0,1)} \log \left(\tilde{h}_{r}^{s}(p)\right) d \mu(p)=\frac{\int_{B_{r}} \log \left(h_{r}^{s}(p)\right) d \omega(p)}{\operatorname{vol}\left(B_{r}\right)} .
$$

To finish the proof it is enough to verify that $\tilde{h}_{r}^{s}(p)$ converges uniformly to 1 and that $\tilde{h}_{r}^{u}(p)$ converges to $h^{u}$ when $r$ goes to 0 . For this, just notice that the bundles $\tilde{E}_{r}^{s}, \tilde{E}_{r}^{c}$ and $\tilde{E}_{r}^{u}$ converge uniformly to $E_{x}, E_{y}$ and $E_{z}$.

2.3. The expanding cocycle. Here we define the actions induced by $f$ and $f_{r}$ on a convenient fiber bundle corresponding to the action on the expanding direction.

We consider the fiber sub-bundle $E_{f}^{u}$ of $T M$ (the unstable bundle of $f$ ), with dimension $d_{u}$. Notice that this sub-bundle is not invariant by $f_{r}$, but is invariant by $f$.

Let $\mathbf{F}_{r}$ be the linear cocycle over $f_{r}$ and acting on $E_{f}^{u}$, such that for any $p \in M$ the linear map $\mathbf{F}_{r}(p): E_{f}^{u}(p) \rightarrow E_{f}^{u}\left(f_{r}(p)\right)$ is defined as follows.

- If $p \notin B_{r} \cup f^{-1}\left(B_{r}\right)$, we just define $\mathbf{F}_{r}(p)=\left(f_{r}\right)_{*}(p)=f_{*}(p)$ : this is possible since $E_{f}^{u}$ is invariant by $f_{*}$.

- If $p \in B_{r}$, then $\mathbf{F}_{r}(p)$ is obtained as follows: first consider $\left(h_{r}\right)_{*}(p)$ acting on $E_{f}^{u}(p)$; its image is some subspace of $T M_{h_{r}(p)}$. We compose $\left(h_{r}\right)_{*}(p)$ with the projection $P^{u}\left(h_{r}(p)\right): T M_{h_{r}(p)} \rightarrow E_{f}^{u}\left(h_{r}(p)\right)$ parallel to $E_{f}^{c s}\left(h_{r}(p)\right)$, getting a linear map from $E_{f}^{u}(p)$ to $E_{f}^{u}\left(h_{r}(p)\right)$. Finally we compose this map with $f_{*}\left(h_{r}(p)\right)$. 
- If $p \in f^{-1}\left(B_{r}\right)$, let $n(p)$ be the smallest positive integer such that $p=f_{r}^{n(p)}(\tilde{q})$ for some $\tilde{q} \in B_{r}$ (being $\infty$ when the negative orbit of $p$ is disjoint from $B_{r}$ ). If $n(p)=+\infty$ we just define $\mathbf{F}_{r}(p)=f_{*}(p)$. If $n(p)$ is finite, we correct the expression by a multiplicative term that corresponds to recovering the centerstable component of $f_{*}^{r}$ that was forgotten by $\mathbf{F}_{r}(\tilde{q})$. More precisely: $\mathbf{F}_{r}(p)=$ $f_{*}(p) \circ P_{r}^{u}(p) \circ\left(f_{r}\right)_{*}^{n(p)}(\tilde{q}) \circ \mathbf{F}_{r}^{-n(p)}(p)$ where $P_{r}^{u}(p)$ is the projection on $E_{f}^{u}(p)$ along $E_{f_{r}}^{c s}(p)$.

We summarize this definition by the following:

$$
\mathbf{F}_{r}(p)= \begin{cases}\left(f_{r}\right)_{*}(p), & \text { if } p \notin B_{r} \cup f^{-1}\left(B_{r}\right), \\ (f)_{*}\left(h_{r}(p)\right) \circ P^{u}\left(h_{r}(p)\right) \circ\left(h_{r}\right)_{*}(p), & \text { if } p \in B_{r}, \\ f_{*}(p) \circ P_{r}^{u}(p) \circ\left(f_{r}\right)_{*}^{n(p)}(\tilde{q}) \circ \mathbf{F}_{r}^{-n(p)}(p), & \text { if } p \in f^{-1}\left(B_{r}\right) .\end{cases}
$$

Since our goal is to understand the behavior of the unstable volume, we also introduce the trivial linear bundle over $M$ whose fibers are $\mathbb{R}$; denote this bundle by $U$. For notational simplicity we write $u(p)$ for the unit element in the fiber of $U$ at the point $p \in M$.

We denote by $F_{r}$ the linear cocycle over $f_{r}$ acting on $U$ as $F_{r}(p)\left(u_{p}\right)=\lambda_{r}(p) u\left(f_{r}(p)\right)$ where $\lambda_{r}(p)$ is the modulus of the determinant of $\mathbf{F}_{r}(p)$.

For any $p$ we denote $\lambda(p)=\left|\operatorname{det} J^{u} f(p)\right|$, and $F_{r}(p, u(p))=\left(f_{r}(p), \lambda_{r}(p)\right.$. $\left.u\left(f_{r}(p)\right)\right)$ where $\lambda_{r}(p)$ is defined by the following.

Remark 2.4. It is possible to write the function $\lambda_{r}(p)$ in the following way:

$$
\lambda_{r}(p)= \begin{cases}\lambda(p), & \text { if } p \notin B_{r} \cup f^{-1}\left(B_{r}\right), \\ \lambda\left(h_{r}(p)\right) h_{r}^{u}(p), & \text { if } p \in B_{r} \\ \lambda(p) A(p), & \text { if } p \in f^{-1}\left(B_{r}\right)\end{cases}
$$

where the function $A(p)$ is the Jacobian of $P_{r}^{u}(p) \circ\left(f_{r}\right)_{*}^{n(p)}(\tilde{q}) \circ \mathbf{F}_{r}^{-n(p)}(p)$.

PROPOSITION 2.5. With the notation previously defined we have

$$
\lim _{r \rightarrow 0} \frac{\sigma_{r}^{u}-\sigma^{u}}{\operatorname{vol}\left(B_{r}\right)}=I(h)<0 .
$$

We devote the rest of this subsection to the proof of this proposition. As a first step we show the next result.

LEMMA 2.6. For any $h C^{1}$-close to identity and any small $r$ one has

$$
\sigma_{r}^{u}=\int_{M} \log \lambda_{r}(p) d \omega(p)
$$

and

$$
\sigma^{u}=\int_{M} \log \lambda(p) d \omega(p) .
$$

Proof. The cocycle is defined in order to have the same exponents of the diffeomorphism, in the same way as in $\S 1$. Since the function $\lambda_{r}(p)$ is integrable then the exponents of the cocycle can again be obtained by integration and the lemma follows. 
From this lemma one deduces the following corollary.

COROLlary 2.7.

$$
\sigma_{r}^{u}-\sigma_{r}=\int_{B_{r}} \log h_{r}^{u}(p) d \omega(p)+\int_{f^{-1}\left(B_{r}\right)} \log A(p) d \omega(p) .
$$

According to Lemma 2.3 we know that

$$
\frac{\int_{B_{r}} \log h_{r}^{u}(p) d \omega(p)}{\operatorname{vol}\left(B_{r}\right)} \rightarrow I(h),
$$

showing that it is sufficient to verify that the other term goes to zero.

LEMMA 2.8.

$$
\lim _{r \rightarrow 0} \frac{\int_{f^{-1}\left(B_{r}\right)} \log A(p) d \omega(p)}{\operatorname{vol}\left(B_{r}\right)}=0 .
$$

Proof. Recall that $A(p)$ is the Jacobian of $P_{r}^{u}(p) \circ\left(f_{r}\right)_{*}^{n(p)}(\tilde{q}) \circ \mathbf{F}_{r}^{-n(p)}(p)$ (see Remark 2.4). In order to estimate this term let us try to understand it in a geometric way.

Writing $\tilde{q}=f_{r}^{-n(p)}(p)$ and $q=h_{r}(\tilde{q})$, one can decompose the linear map

$$
\left(h_{r}\right)_{*}(\tilde{q}): E_{f}^{u}(\tilde{q}) \rightarrow T_{q} M=E_{f}^{u}(q) \oplus E_{f}^{c s}(q)
$$

as $\left(h_{r}\right)_{*}(\tilde{q})=\left(H_{r}^{u}(\tilde{q}), V_{r}(\tilde{q})\right)$ where $V_{r}(\tilde{q})$ is a linear map from $E_{f}^{u}(\tilde{q})$ to $E_{f}^{c s}(q)$ and where $H_{r}^{u}(\tilde{q})$ is the linear map defined in the paragraph before Lemma 2.3 and whose Jacobian is $h_{r}^{u}(\tilde{q})$.

Consider now $\left(f_{r}\right)_{*}^{n(p)}(\tilde{q}): E_{f}^{u}(\tilde{q}) \rightarrow T_{p} M$. One decomposes the tangent space $T_{p} M$ as $T_{p} M=E_{f}^{u}(p) \oplus E_{f}^{c s}(p)$. In this decomposition, $\left(f_{r}\right)_{*}^{n(p)}(\tilde{q})$ can be written as

$$
\left(f_{r}\right)_{*}^{n(p)}(\tilde{q})=\left(\mathbf{F}_{r}^{n(p)}(\tilde{q}), f_{*}^{n(p)}(q) \circ V_{r}(\tilde{q})\right): E_{f}^{u}(\tilde{q}) \rightarrow T_{p} M .
$$

So we get

$$
\left(f_{r}\right)_{*}^{n(p)}(\tilde{q}) \circ \mathbf{F}_{r}^{-n(p)}(p)=\left(\operatorname{Id}, f_{*}^{n(p)}(q) \circ V_{r}(\tilde{q}) \circ \mathbf{F}_{r}^{-n(p)}(p)\right) .
$$

Recall that $P_{r}^{u}(p)$ denotes the projection of $T_{p}(M)$ on $E_{f}^{u}(p)$ along the center stable space $E_{f_{r}}^{c s}(p)$ of $f_{r}$ at $p$. So $A_{r}(p)$ is the Jacobian of

$P_{r}^{u}(p) \circ\left(\operatorname{Id}, f_{*}^{n(p)}(q) \circ V_{\tilde{q}} \circ \mathbf{F}_{r}^{-n(p)}(p)\right)=\operatorname{Id}+\left(P_{r}^{u}(p) \circ f_{*}^{n(p)}(q) \circ V_{r}(\tilde{q}) \circ \mathbf{F}_{r}^{-n(p)}(p)\right)$.

One denotes $\mathcal{A}_{r}(p)=P_{r}^{u}(p) \circ f_{*}^{n(p)}(q) \circ V_{r}(\tilde{q}) \circ \mathbf{F}_{r}^{-n(p)}(p): E_{f}^{u}(p) \rightarrow E_{f}^{u}(p)$. Using this notation, one has $A_{r}(p)=\operatorname{det}\left(\operatorname{Id}+\mathcal{A}_{r}(p)\right)$.

LEMMA 2.9. There exists $C>0$ and $\alpha \in] 0,1[$ such that, for any small $r>0$ and any $p \in f^{-1}\left(B_{r}\right)$, we have $\left\|\mathcal{A}_{r}(p)\right\| \leq C \alpha^{n(p)}$.

We postpone the proof of this lemma and now we finish the proof of Lemma 2.8. Lemma 2.9 asserts that $\mathrm{Id}+\mathcal{A}_{r}(p)$ goes uniformly to Id as $r \rightarrow 0$, and so $A_{r}(p)$ goes uniformly to 1 as $r \rightarrow 0$; using the fact that $\operatorname{vol}\left(f^{-1}\left(B_{r}\right)\right)=\operatorname{vol}\left(B_{r}\right)$ we obtain:

$$
\lim _{r \rightarrow 0} \frac{\int_{f-1\left(B_{r}\right)} \log A(p) d \omega(p)}{\operatorname{vol}\left(B_{r}\right)}=0,
$$

as claimed. 
Proof of Lemma 2.9. First notice that the norm of the projection $\tilde{P}_{p}^{u}: T_{p} M \rightarrow E_{f}^{u}(f)$ along $E_{f_{r}}^{c s}(p)$ is uniformly bounded in $r$ and $p$; for this, it is enough to see that the angle $L\left(E_{f_{r}}^{c s}, E_{f}^{u}\right)$ is greater than some constant $C_{1}$, because the bundle $E_{f_{r}}^{c s}$ is close to $E_{f}^{c s}$.

Then notice that the norm $\left\|V_{\tilde{q}}\right\|$ is also uniformly bounded by some constant $C_{2}$ since $h_{r}$ is $C^{1}$-close to the identity and $V_{\tilde{q}}$ is obtained by composing the differential $\left(h_{r}\right)_{*}(\tilde{q})$ with the projection on $E_{f}^{c s}(q)$ along $E_{f}^{u}(q)$.

Consider now the linear map $\mathbf{F}_{r}^{-n(p)}(p): E_{f}^{u}(p) \rightarrow E_{f}^{u}(\tilde{q})$. This map is obtained by composing the differential $f_{*}^{-n(p)}(p): E_{f}^{u}(p) \rightarrow E_{f}^{u}(q)$ with the linear map $\left(H_{r}^{u}(q)\right)^{-1}: E_{f}^{u}(q) \rightarrow E_{f}^{u}(\tilde{q})$ (defined in the paragraph before Lemma 2.3). Notice that the norm of $\left(H_{r}^{u}(q)\right)^{-1}$ is uniformly bounded (in $r$ and $q \in B_{r}$ ) by some constant $C_{3}$.

Using these three inequalities, we want to bound the norm of $\mathcal{A}_{r}(p)=P_{r}^{u}(p) \circ$ $f_{*}^{n(p)}(q) \circ V_{r}(\tilde{q}) \circ \mathbf{F}_{r}^{-n(p)}(p): E_{f}^{u}(p) \rightarrow E_{f}^{u}(p)$. For this, consider a unit vector $w \in E_{f}^{u}(p)$. Then $w_{1}=\left(\mathbf{F}_{r}^{-n(p)}(p)\right)(w)$ is a vector of $E_{f}^{u}(\tilde{q})$ whose norm is bounded by $C_{3}\left\|\left(f_{*}^{-} n(p)(p)\right)_{E_{f}^{u}(p)}\right\|$, where $\left(f_{*}^{-n(p)}(p)\right)_{E_{f}^{u}(p)}$ denotes the restriction to $E_{f}^{u}(p)$ of the linear map $f_{*}^{-n(p)}(p)$. So $w_{2}=V_{r}(\tilde{q})\left(w_{1}\right)$ is a vector of $E_{f}^{c s}(q)$ whose norm is bounded by $C_{2} C_{3}\left\|\left(f_{*}^{-n(p)}(p)\right)_{E_{f}^{u}(p)}\right\|$. Finally $\mathcal{A}_{r}(p)(w)=\left(P_{r}^{u}(p) \circ f_{*}^{n(p)}(q)\right)\left(w_{2}\right)$ is a vector of $E_{f}^{u}(p)$ whose norm is bounded by

$$
\left\|\mathcal{A}_{r}(p)(w)\right\| \leq C_{1} C_{2} C_{3}\left\|\left(f_{*}^{n(p)}(q)\right)_{E_{f}^{c s}(q)}\right\|\left\|\left(f_{*}^{-n(p)}(p)\right)_{E_{f}^{u}(p)}\right\|,
$$

where $\left(f_{*}^{n(p)}(q)\right)_{f}^{c s}(q)$ denotes the restriction to $E_{f}^{c s}(q)$ of the map $f_{*}^{n(p)}(q)$.

Finally, by definition of the domination, there are constants $C_{4}>0$ and $\left.\alpha \in\right] 0,1[$ such that for any point $x \in M$, any integer $n \in \mathbb{N}$ and any unit vectors $w_{1} \in E_{f}^{u}(x)$ and $w_{2} \in E_{f}^{c s}(x)$, one has $\left\|f_{*}^{n}\left(w_{1}\right)\right\| \leq C_{4} \alpha^{n}\left\|f_{*}^{n}\left(w_{2}\right)\right\|$. As a consequence,

$$
\left\|\left(f_{*}^{n(p)}(q)\right)_{E_{f}^{c s}(q)}\right\|\left\|\left(f_{*}^{-n(p)}(p)\right)_{E_{f}^{u}(p)}\right\| \leq C_{4} \cdot \alpha^{n(p)} .
$$

So the lemma follows by choosing $C=C_{1} C_{2} C_{3} C_{4}$.

2.4. The contracting cocycle. We keep the notation of the last subsection. Now we consider the sub-bundle $E_{f}^{s}$ of $T M$ invariant by $f^{-1}$ and again we use a trivial bundle $S$ (analogous to $U$ ) in order to control the effect of the perturbation on the contracting components. It is possible to write the perturbation as $g_{r}=f \circ h_{r}^{-1} \circ f^{-1}$, with support on $f(B)$. Choosing a Riemannian metric on $f\left(B_{r}\right)$ that makes $f$ an isometry we can write the corresponding cocycle as in the last section, just replacing $f$ by $f^{-1}$ and $h_{r}$ by $g_{r}$. With this definition it is possible to verify that the sum of the stable Lyapunov exponents of the diffeomorphism is the same as the sum of the exponents of the cocycle and we can write the difference between the perturbed and the unperturbed cases as a difference of integrals. Again, from Lemma 2.3 we get that

$$
\lim _{r \rightarrow 0} \frac{\sigma_{r}^{s}-\sigma^{s}}{\operatorname{vol}\left(B_{r}\right)}=0
$$

showing that the perturbation almost does not affect the sum of the exponents in $E^{s}$.

This result together with the estimate of the perturbation in the expanding direction (Proposition 2.5) ends the proof of Proposition 2.1: the action of the pertubation on the sum of the integrated expanding exponents is greater than the action on the sum of the integrated contracting exponents. 
2.5. Concluding the proof. Since the diffeomorphism preserves the volume measure $\omega$ and then the sum of all exponents must be zero, it follows from the results of the last subsections that the difference between the sum of the integrated center exponents for $f_{r}$ and the sum of the integrated center exponents for $f$ is different from zero, as claimed (this ends the proof of Theorem 1, Theorem 4 and Proposition 0.3).

Notice that the perturbed diffeomorphism $f_{r}$ is $f_{r}=f \circ h_{r}$ where $h_{r}$ can be chosen smooth: if $f$ is $C^{r}$, the perturbation $f_{r}$ is $C^{r}$ too.

Finally, the proof of Theorem 2 follows exactly from the same argument: consider a volume-preserving diffeomorphism $f$ admitting a dominated splitting $E_{1} \oplus \cdots \oplus E_{k}$. Then, for any $1<i<k$, one can build a volume-preserving $C^{1}$-perturbation $f_{r}^{i}$ of $f$ such that, for any small $r$, the action of the perturbation on the sum of the integrated exponents corresponding to $E_{i+1} \oplus \cdots \oplus E_{k}$ is greater than the action on the sum of the integrated exponents corresponding to $E_{1} \oplus \cdots \oplus E_{i-1}$. As a consequence, the perturbation $f \rightarrow f_{r}^{i}$ increases the sum of the integrated exponents corresponding to $E_{i}$.

Acknowledgements. This work has been supported by the French Ministère des Affaires Étrangères through the Franco-Brasilian Cooperation in Mathematics. We thank the Laboratoire de Topologie Dijon for its warm hospitality. We thank the referee for his/her careful reading of the manuscript and his/her patience with our knowledge of English.

\section{REFERENCES}

[Boc] J. Bochi. Genericity of zero exponents. Ergod. Th. \& Dynam. Sys. 22 (2002), 1167-1696.

[BocVi] J. Bochi and M. Viana. Uniform (projective) hyperbolicity or no hyperbolicity: a dichotomy for generic conservative systems. Annales Inst. Henri Poincaré-Analyse Non-Linéaire 19 (2002), $113-123$.

[BoGVi] C. Bonatti, X. Gomez-Mont and M. Viana. Généricité d'exposants de Lyapunov non-nuls pour des produits déterministes de matrices. Annales Inst. Henri Poincaré-Analyse Non-Linéaire 20(4) (2003), 579-624.

[BoVi] C. Bonatti and M. Viana. Lyapunov exponents with multiplicity 1 for deterministic products of matrices. Preprint, Université de Bourgogne, 2001.

[DoWi] D. Dolgopyat and A. Wilkinson. Stable accessibility is $C^{1}$ dense. Astérisque to appear.

[FHY] A. Fathi, M. Herman and J. C. Yoccoz. A proof of Pesin's stable manifold theorem. Geometric Dynamics (Rio de Janeiro, 1981) (Lecture Notes in Mathematics, 1007). Springer, Berlin, 1983, pp. 177-215.

[HPS] M. Hirsch, C. Pugh and M. Shub. Invariant Manifolds (Lecture Notes in Mathematics, 583). Springer, Berlin, 1977.

[ShWi] M. Shub and A. Wilkinson. Pathological foliations and removable zero exponents. Invent. Math. 139(3) (2000), 495-508. 